\title{
The Effeciency of Cryotop in Vitrification of In Vitro Produced Egyptian Buffalo
} (Bubalus Bubalis) Embryos El-Sayed, A. ${ }^{1,2}$; A. Gad ${ }^{1,2}$; M. AboelEla ${ }^{1,2}$ and G. Ashour ${ }^{1}$

${ }^{1}$ Department of Animal Production, Faculty of Agriculture,Cairo University,12613, Giza, Egypt.

${ }^{2}$ Embryology and Cell Culture Research Laboratory, Cairo University Research Park (CURP), Faculty of Agriculture, 12613 Giza, Egypt.

\begin{abstract}
Embryo cryopreservation becomes a pivotal side in the shape of assisted reproductive technologies. It plays an essential role in several cases such as, embryo storing during transportation, embryo preservation for future usesand establishment ofcryobanks for endangered species and rare breeds. The presentexperiment was conducted to evaluate the cryotoleranceofin vitro produced buffalo embryosat blastocyst stage to vitrification process using cryotop. Embryos were produced using conventional in vitro fertilisation technique and had been divided into two categories: non-vitrified group, which was culturedin vitro till hatched blastocyst stage and vitrified group, which was collected at day 7 for cryopreservation. A stepwise vitrification and thawing procedures were performed using Cryotop. Survival rate of embryos was observed within 4 hours of thawing, then the ability to expand and hatch was recorded.The expansion rate was higher in control group compared to vitrified group (91.66 vs. $86.36 \%$, respectively) with no significant differences. In both groups, all expanded blastocysts reached the hatching stage normally.Therefore, our results suggested thatCryotop is aneffective tool for cryopreservation of in vitro produced Egyptian buffalo blastocyst.
\end{abstract}

Keywords: Buffalo; Embryo; Vitrification; Cryotop; In vitro fertilisation.

\section{INTRODUCTION}

Water buffalo is considered to be major milk and meat producing livestock in many developing counties, especially Egypt. Although buffalo can adapt to difficult environmental conditions, it has a low reproductive efficiency. Water buffalo has not received the same concern from genetic improvement and applying reproductive technologies, as cattle. Many of these modern techniques, which have been successfully applied on bovine, could effectively help in overcoming the low reproductive efficiency of buffalo that compromises its performance.

The use of in vitro embryo production technology could significantly enhance the efficiency and logistics of embryo production in water buffalo (Drost, 2007). For example, taking the advantage of using in vitro embryo production techniques will help in exploiting wasted geneticallyvaluable oocytes of slaughtered females. In buffalo, there were limited successful uses of in vitro embryo production techniques with low yield of high quality transferable embryos.Obviously, The system of laboratory production of buffalo embryos still sub-optimal and needs more considerable improvements (Nandi et al., 2002).

Superovulation and embryo transfer in buffalo have poor results (Drost, 2007). However, cryopreservation could be a beneficial way to preserve embryos and other germ cells, as a genetic resource, till a significant development would be achievedin these techniques. Unfortunately, there is a lake in research experiments conducted on buffalo embryos to evaluate different methods of cryopreservation. Therefore, it's difficult to choose the proper technique that leads to the best survival, pregnancy and live calves production rate.

Since 1984, when Fahy and his colleagues published the first description of their vitrification approach as a new method for cryopreservation, vitrification has increasingly and rapidly used and proposed to became the most suitable method for cryopreservation of any cells or tissues in the near future (Fahy and Rall, 2007).
Vitrification, a type of cryopreservation techniques,is a process of glass solidification of a liquid by increasing its viscosity to become in vitreous structure (Craig and Turner, 2013). There are two main cryopreservation methods: the slow freezing method, which needs sophisticated instrument; takes long time and could not eliminate cryoinjuries completely,andthe vitrification method a cheap, simple and rapid technique, and appeared to be the effective alternative (Parnpaiet al., 2016).

Vitrification depends on using high concentration of cryoprotective agent (CPA) and sharply cooling and warming rate. Cryoprotectent agent is a chemical compoundused to protect the cell from cryoinjuries by regulating water dynamics during cooling. Cryoprotectents are grouped into two major categories, working together: penetrating agents, which can cross cell membrane and replace the intracellular water content and lowering the freezing temperature to prevent ice crystal formation, and non-penetrating agents, which has a large mass to diffuse into cells so they work on cellular dehydration by increasing osmolarity of the extracellular space. The most common penetrating CPAs are a mixture of Dimethyl sulfoxide (DMSO) and Ethylene glycol (EG) with different concentration, which minimise the cytotoxicity effect of the CPA, and sucrose is considered to be the most frequent used non-penetrating CPAs (Swain and Smith, 2010).

The Cryotop was invented specifically in order to improve the cryopreservation of human embryos. This method,which is considered a minimum-volume cooling procedure, consists of loading the embryos on a veryfine polypropylene strip using an extremely small volume less than $0.01 \mu 1$ of vitrification solution and immediately plungethem into liquid nitrogen. In the presence of the CPA, The reduction ofthe amount ofvitrification medium surrounding the embryos allowsthem to rapidly pass the critical temperature zone. Those changesare the main factors responsible for the 
best results obtainedwith this approach for several species (Morimoto, 2010 and Leme, 2016).

Therefore, the objective of this study was to evaluate the cryotoleranceof in vitro produced buffalo embryosat blastocyst stage to vitrification technique using Cryotop.

\section{MATERIALS AND METHODS}

Unless otherwise indicated, chemicals were purchased from Sigma-Aldrich (St. Louis, Missouri, USA).

\section{Experimental Design:}

A total number of 48 buffalo blastocysts (day 7) were produced in vitrofrom ten replicates and randomly distributed into twoequal groups. The first group (24 blastocysts) was vitrified using Cryotop device and thawed, while the second group (24 blastocysts) was cultured in vitro as fresh embryos (control group).Both groups continued culturein vitro till hatching.

Collection, Transportation and Preparation of ovaries:

Buffalo ovaries were collected from slaughtered buffalos fromthree different local abattoirs and transported to the lab within $2 \mathrm{hrs}$ in a thermos containing sterilised physiological saline $(0.9 \% \mathrm{NaCl})$ supplemented with antibiotic $(100 \mu \mathrm{g} / \mathrm{ml}$ streptomycin sulphate and $100 \mathrm{IU} / \mathrm{ml}$ penicillin) and maintained at a temperature of $30-35^{\circ} \mathrm{C}$. The collected ovaries were washed once in sterilised physiological saline, then once in $70 \%$ ethanol alcohol and twice in sterilised physiological saline again.

Oocytes recovery:

Cumulus oocyte complexes (COCs) were aspirated from 2-8mm diameter follicles using 18-gauge needle attached to a $10 \mathrm{ml}$ syringe. COCs received in pre-warmed Hepes-buffered medium 199 (Gibco, UK). Using stereomicroscope, COCs with homogeneous cytoplasm and surrounded by at least three layers of cumulus cells were selected as good quality oocytes.

\section{In vitro maturation:}

Selected COCs were washed three times by gently pipetting in pre-warmed maturation media (modified Parker medium supplemented with 12\% inactivated foetal bovine serum and $10 \mu \mathrm{g} / \mathrm{ml} \mathrm{FSH}$ ). Maturation was performed in 4-well plate (Nunc, Denmark), where 30 COCs were incubated in $400 \mu$ of maturation media over lied with $400 \mu 1$ of mineral oil for 18 hours at $38.5^{\circ} \mathrm{C}$ in a humidified atmosphere containing $5 \% \mathrm{CO}_{2}$.

\section{Semen preparation:}

Frozen $0.25 \mathrm{ml}$ semen straw was thawed in water bath at $37^{\circ} \mathrm{C}$ for 30 seconds, and then wiped with $70 \%$ ethanol alcohol before being opened. Sperm washed three times by centrifugation at $500 \mathrm{xg}$ for 5 minutes, twice in sperm washing medium (modified $\mathrm{Ca}^{2+}$ free TALP medium) and the last one in fertilisation medium (modified TALP medium). The final pellet was re-suspended in fertilisation medium and the final concentration of sperm was adjusted to be $2 \times 10^{6} / \mathrm{ml}$.

In vitro fertilisation:

Conventional IVF was performed in 4-well plates, where each 30 matured oocytes were co-cultured with the prepared sperm in $400 \mu 1$ of fertilisation medium over lied with $400 \mu 1$ of mineral oil for 18 hours at $38.5^{\circ} \mathrm{C}$ in a humidified atmosphere with $5 \% \mathrm{CO}_{2}$.

\section{In vitro culture:}

Presumed zygotes were transferred to embryo washing medium (modified Hepes-SOF medium), and mechanically denuded by repeated pipetting. Denuded zygotes were cultured, using 4-well plates, in culture medium (modified SOFAA medium) for 7 days at $38.5^{\circ} \mathrm{C}$ in a humidified atmosphere with $5 \% \mathrm{CO}_{2}, 5 \%$ $\mathrm{O}_{2}$, and $90 \% \mathrm{~N}_{2}$. Culture medium was half renewed every 48 hours.

Vitrification:

Blastocysts at day 7 were vitrified using Cryotop (Kitazato, Japan). Groups of three blastocysts were equilibrated in holding medium for 5 minutes (Hepes-buffered medium 199 supplemented with 20\% FBS and $50 \mathrm{~g} / \mathrm{ml}, 1 \mathrm{M}$ DMSO and $7.5 \%$ EG). Equilibrated blastocysts were transferred to the vitrification solution (Hepes-buffered medium 199 supplemented with $20 \% \mathrm{FBS}$ and $50 \mathrm{~g} / \mathrm{ml}, 0.5 \mathrm{M}$ sucrose, 2 M DMSO and 15\% EG) for 30 seconds. All vitrification procedures were performed at room temperature. Each group was then loaded on a Cryotop with less than $0.1 \mu 1$ of vitrification solution and plunged into liquid nitrogen $\left(\mathrm{LN}_{2}\right)$ and stored for 6 months.

Thawing/Warming:

Cryotop was directly inserted into $1 \mathrm{ml}$ of warming medium (Hepes-buffered medium 199 supplemented with $20 \%$ FBS and $0.5 \mathrm{M}$ sucrose) at $37^{\circ} \mathrm{C}$ for 1 minute. Blastocysts were then sequentially rehydrated in the warming mediumat $37^{\circ} \mathrm{C}$ in stepwise serial dilution of $0.25,0.1$ and $0 \mathrm{M}$ sucrose for 5,5 and 10 minutes, respectively. Post-warming, all thawed blastocysts were subjected to in vitro culture.

\section{Statistical analysis:}

Data were analysed using the Statistical Analysis System (SAS), University Edition (SAS Institute Inc., Cary, NC, USA). Differences in mean values were tested using analysis of variance followed by t-test for two independent means. Differences of $\mathrm{P} \leq 0.05$ were considered to be significant.

\section{RESULTS AND DISCUSSION}

The objective of this study was to evaluate the cryotolerance of in vitro produced buffalo blastocyst to vitrification technique using Cryotop. Embryos were produced using conventional in vitro fertilisation technique. In general, cleavage rate was $61.32 \%$ and theaverage blastocyst rate (Day 7) (Fig. 2), calculated based on the cleaved embryos,was $27.27 \%$. Only embryos in the blastocyst stage were usedfor vitrification. After thawing, embryos with cryoinjuries like zona cracking and shrinkagewere considered to be non-survived (Fig. 1). Blastocyst expansion and hatching rates were calculated for both groups. Those blastocysts that re-expanded were considered alive.In the vitrified group, only 2 blastocysts out of the 24 were appeared morphologically abnormal after the vitrification process and considered as non-survived embryos. The remaining blastocysts were survived and continued development (Fig. 2). The expansion rate was 
higher in control group compared to vitrified group ( 91.66 vs. $86.36 \%$, respectively) with no significant differences (Table 1). In both groups, all expanded blastocysts reached the hatching stage normally.

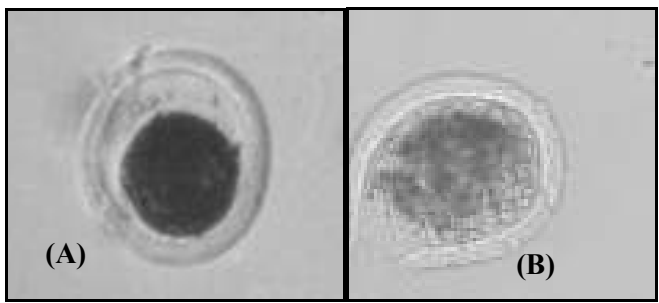

Figure 1. Non-survived blastocyst after warming: (A) shrinkage and (B) zona cracking.

In an experiment conducted in China(Yang et al., 2012) to evaluate the cryotolerance of IVF produced blastocysts using open-pulled straw (OPS) and slaw freezing in $0.25 \mathrm{ml}$ French straw, the survival rate of embryos vitrified in three different concentrations of cryoprotectent (40\% EG, 25\% EG $+25 \%$ DMSO and $20 \% \mathrm{EG}+20 \% \mathrm{DMSO}$ ) were $63.1 \%, 78.0 \%$ and $88.7 \%$, respectively. On the other hand, the cryosurvival of slow freezing in two different concentrations $(10 \% \mathrm{EG}$

Table 1. Survival, expansion and hatching rates from vitrified and non-vitrified (control) blastocysts using the Cryotop method.

\begin{tabular}{lcccccc}
\hline Groups & $\begin{array}{c}\text { Immature } \\
\text { oocytes }\end{array}$ & Cleavage & $\begin{array}{c}\text { Blastocyst } \\
\text { (day 7)No. }\end{array}$ & $\begin{array}{c}\text { Survival rate } \\
\text { No. (\%) }\end{array}$ & $\begin{array}{c}\text { Expansion rate } \\
\text { (24 hours) } \\
\text { No. (\%) }\end{array}$ & $\begin{array}{c}\text { Hatching rate } \\
(\mathbf{4 8} \text { hours) } \\
\text { No. (\%) }\end{array}$ \\
\hline Vitrified & 150 & 89 & 24 & $22(91.66)$ & $19(86.36)$ & $19(86.36)$ \\
Control & 150 & 87 & 24 & $24(100)$ & $22(91.66)$ & $22(91.66)$ \\
\hline
\end{tabular}

The effect of vitrification of in vitro derived blastocyst using $0.25 \mathrm{ml}$ straw has been studied by (Hufana-Duranet al., 2004) and found that hatched blastocyst rate after thawing was $75 \%$. Another study showed that the blastocyst hatching rate after vitrification using $0.25 \mathrm{ml}$ straw of in vitro derived blastocyst using oocytes aspirated from slaughterhouses ovaries was $40.2 \%$ versus $52.8 \%$ in oocytes were collected by OPU from live animals(Manjunathaet al., 2008).

In Bovine, most of studies that used Cryotop were conducted on oocytes, however, some studies used Cryotop to cryopreservedembryos at different stages (day 5, 6 and 7) and the survival ratesof the vitrified embryos were $96.5 \%, 87.5 \%$ and $94.2 \%$, and hatched embryos rates were $32.7,52.4 \%$ and $67.3 \%$, respectively(Kellyet al., 2003).Lemeet al., (2016) vitrified in vitro produced blastocyst (day between 6 and 6.5 ) and survival, re-expansion and expanded blastocyst rates were $87 \%, 58.7 \%$ and $28.4 \%$, respectively. When Cryotop was compared with aluminium block and conventional cryopreservation of in vitro produced bovine blastocyst at day 7 the survival rates were $85.9 \%, 70.6 \%$ and $56.1 \%$, respectively (Kruse, 2012). Inabaet al., (2011) investigated the effect of vitrification by Cryotop on re-expansion, hatching and hatched rate after thawing of cryopreserved day 6 blastocyst and they were $100 \%, 93.2 \%$ and $95.5 \%$, respectively.

Generally, Cryopreservation of embryos would be affected by many factors adversely or supportively in and $0.05 \mathrm{M}$ trehalose dehydrate $+1.8 \% \mathrm{EG}+0.4 \% \mathrm{BSA}$ ) were $75.2 \%$ and $64.6 \%$.

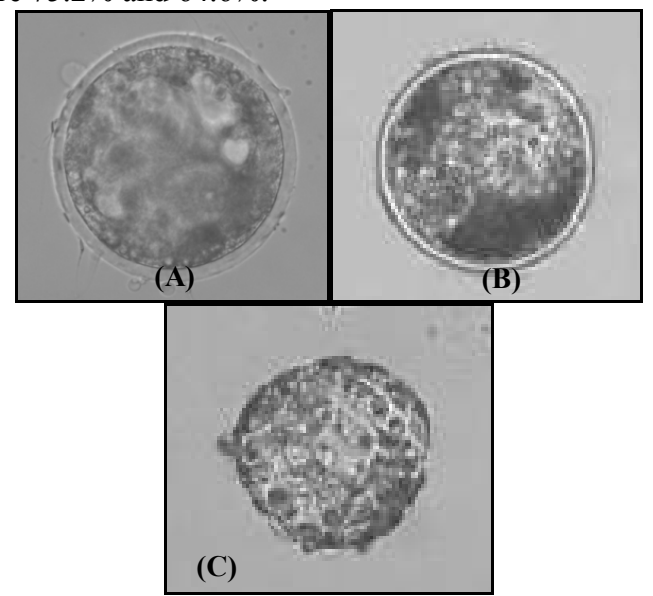

Figure 2. Blastocyst morphology before and after warming: (A) day 7 blastocyst which was used in vitrification, (B) re-expanded blastocyst after 24 hours of warming and (C) hatched blastocyst after 48 hours of warming.

different pitches. One of these factors which influence the cryosurvival of embryos is the CPA. Using of mixture of cryoprotectents such as EG and DMSO have some advantage over using only one type. The combining of various cryoprotectent enable the use of lower concentration of each compound in vitrification solution and resulting in alleviating the toxicity (Manjunatha et al., 2008). In addition, using CPAs at room temperature or less than $37^{\circ} \mathrm{C}$ could be a reason of decreasing their toxicity (Kuwayamaet al., 2015). Furthermore, using stepwise addition and removal of cryoprotective agents upon warming/ thawing by gradually increasing and decreasing the CPA concentration help in minimising the osmotic stress(Swain and Smith, 2010).

Kuwayamaet al., (2005) mentioned that when Cryotop was used, the cooling rate was $22800^{\circ} \mathrm{C} /$ minute, warming rate was $42100^{\circ} \mathrm{C} /$ minute and volume of solution was less than $0.1 \mu \mathrm{l}$. Reducing the volume of the sample and increasing the cooling rate promotes vitrification by decreasing the amount of liquid which has to be cooled and declining the probability of ice crystal formation (Yavin and Arav, 2007).

By comparing the obtained results in the current study with the previous experiments in which vitrification method was used to cryopreserve in vitro produced embryos, it seems that Cryotop device has a superior efficiency more than other used tools. Regardless of using different CPA concentration and 
exposure time, the successful of vitrification using Cryotop could be attributed to three main factors: using a moderate concentration of mixed CPAs, a minimal volume of vitrification solution and ultra-rapid cooling and warming rates.

\section{CONCLUSION}

In conclusion, this study demonstrated that vitrification method using Cryotopallows acceptable survival and development rates of in vitro produced blastocyst in Egyptian buffalo. These results showed promising information for usingCryotop in vitrification method for preservation of in vitro produced embryos in buffalo.

\section{REFERENCES}

Craig, J. and Turner, K.(2013).Cryopreservation in assisted reproduction.In:Text book of clinical embryology. (Eds. Coward, K. and Wells, D.). Cambridge University Press, Cambridge, pp.327-336.

Do, V.H.; Walton S. and Taylor-Robinson A.W.(2014).Benefits and constraints of vitrification technologies for cryopreservation of bovine in vitro fertilized embryo. Journal of Veterinary Science and Animal Husbandry, 2(4): 401.

Drost, M.(2007).Advancedreproductive technology in the water buffalo. Theriogenology, 68:450-453.

Fahy, G.M. and Rall, W.F. (2007). Vitrification: an overview. In: Vitrification in assisted reproduction. (Eds. Tucker, J. and Liebermann, J.).Informa Healthcare, London, pp. 1-20.

Hufana-Duran, D.; Pedro, P.B.; Venturina, H.V.; Hufana R.D.; Salazar, A.L.; Duran, P.G. and Cruza, L.C. (2004). Post-warming hatching and birth of live calves following transfer of in vitro-derived vitrified water buffalo (Bubalusbubalis) embryos. Theriogenology, 61:1429-1439.

Inaba, Y.; Aikawa, Y.; Hirai, T.; Hashiyada, Y.; Yamanouchi, T.; Misumi, K.; Ohtake, M.; Somfai, T.; Kobayashi, S.; Saito, N.; Matoba, S.; Konishi, K. and Imai, K. (2011). In-straw Cryoprotectant Dilution for Bovine Embryos Vitrified Using Cryotop. Journal of Reproduction and Development, 57(4):437-43.

Kelly, J.M.; Kleemann, D.O.; Kuwayama, M. and Walker, S.K.(2003).Vitrification of in vitro-produced bovine and ovine embryos using the minimum volume cooling Cryotop method. Reproduction Fertility and Development, 16(2):172-173.
Kruse, S. (2012).Vitrification of in vitro- and in vivoproduced bovine embryos for direct transfer.Master thesis, Department of Biomedical Sciences, Colorado State University, Colorado.

Kuwayama, M.; Gandhi, G.; Kagalwala, S. and Ramani, R.(2015). Vitrification: An Overview. In: Vitrification in Assisted Reproduction A User's Manual. (Eds. Allahbadia, G.; Kuwayama, M. and Gandhi, G.).Springer, New Delhi, pp.1-7.

Kuwayama, M.; Vajta, G.; Kato, O. and Leibo, S.P. (2005).Highly efficient vitrification method for cryopreservation of human oocytes. Reproductive BioMedicine Online, 3(11):300-308

Leme, L. de O.;Dufort, I.;Spricigo, J.F. W.;Braga, T.F.; Sirard, M.; Dode, M.A.N. and Franco M.M. (2016). Effect of vitrification using the Cryotop method on the gene expression profile of in vitro-produced bovine embryos. Theriogenology, 85:724-733.

Manjunatha, B.M.; Gupta, P.S.P.; Ravindra, J.P.; Devaraj, M. and Nandi, S. (2008).In vitro embryo development and blastocyst hatching rates following vitrification of river buffalo embryos produced from oocytes recovered from slaughterhouse ovaries or live animals by ovum pick-up. Animal Reproduction Science, 104:419-426.

Morimoto, Y. (2010).Cryopreservationof blastocysts. In: Fertility cryopreservation (Eds. Chian, R. and Quinn, P.).Cambridge University Press, Cambridge, pp. 95105.

Nandi, S., Raghu, H.M., Ravindranatha, B.M. and Chauhan, M.S. (2002). Production of Bu $\square$ alo (Bubalusbubalis) Embryos in vitro: Premises and Promises. Reproduction in Domestic Animals, 37, 65-74.

Parnpai, R.; Liang, Y., Ketudat-Cairns, M., Somfai, T. and Nagai, T. (2016).Vitrification of buffalo oocytes and embryos. Theriogenology, 86(1):214-220.

Perera, B.M.A.O. (2011). Reproductive cycles of buffalo. Animal Reproduction Science, 124:194-199.

Swain, J. E. and Smith,G. D.(2010).Cryoprotectants.In: Fertility cryopreservation (Eds. Chian, R. and Quinn, P.)Cambridge University Press,Cambridge, pp.16-23.

Yang, C.Y.; Pang, C.Y.; Yang, B.Z.; Li, R.C.; Lu, Y.Q. and Liang, X.W. (2012).Optimization of cryopreservation of buffalo (Bubalusbubalis) blastocysts produced by in vitro fertilization and somatic cell nuclear transfer.Theriogenology, 78(7):1437-1445.

Yavin, S. and Arav, A. (2007).Measurement of essential physical properties of vitrification solutions. Theriogenology, 67:81-89.

\footnotetext{
كفاءة إستخدام الـ Cryotop في تجميد أجنة الجاموس المصري المنتج معمليًا.

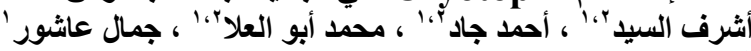

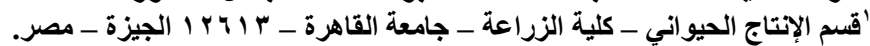

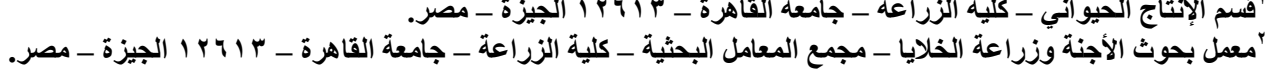

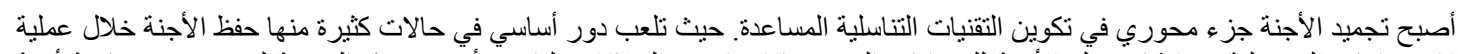

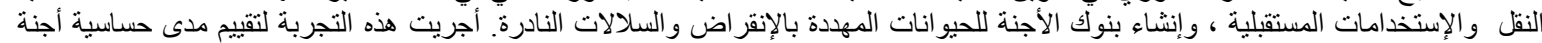

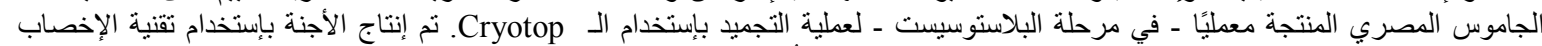

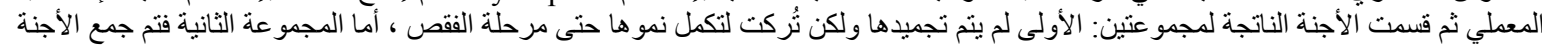

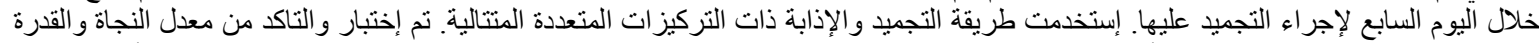

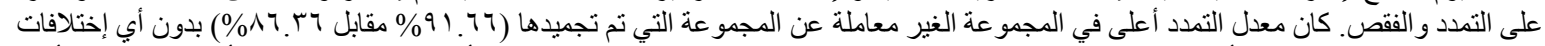

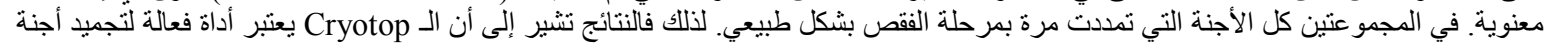

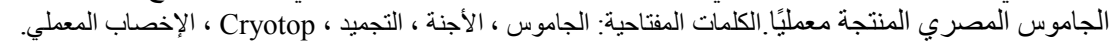

